\title{
Measurements of annual parallaxes and proper motions of the red supergiant S Per
}

\author{
Yoshiharu Asaki ${ }^{1}$, Shuji Deguchi ${ }^{2}$, Hirishi Imai ${ }^{3}$, Kazuya Hachisuka ${ }^{4}$, \\ Makoto Miyoshi ${ }^{5}$ and Mareki Honma ${ }^{5}$ \\ ${ }^{1}$ ISAS, 3-1-1 Yoshinodai, Sagamihara, 229-8510, JAPAN \\ email: asaki@vsop.isas.jaxa.jp \\ ${ }^{2}$ Nobeyama Radio Observatory, Nobeyama, Minamimaki, Minamisaku, 384-1305, JAPAN \\ ${ }^{3}$ Kagoshima University, 1-21-24 Korimoto, Kagoshima, 890-8580, JAPAN \\ ${ }^{4}$ Shanghai Astronomical Observatory, Chinese Academy of Sciences, Shanghai, 200030, CHINA \\ ${ }^{5}$ National Astronomical Observatory, 2-21-1, Osawa, Mitaka, 181-8588, JAPAN
}

\begin{abstract}
VLBI phase-referencing monitoring of water vapor masers around the red supergiant, S Per, was conducted over four years. We successfully obtained proper motions and an annual parallax of the masers and determined the distance to S Per of $2.51 \pm 0.09 \mathrm{kpc}$. The proper motion of the star itself was inferred from the maser proper motions, and it was -0.38 and $-1.54 \mathrm{mas} / \mathrm{yr}$ for right ascension and declination, respectively. Assuming the distance from the sun to the Galactic center, $R_{0}$, of $8.5 \mathrm{kpc}$ and the rotation velocity around the sun, $\Theta_{0}$, of $220 \mathrm{~km} / \mathrm{s}$, the Galactic rotation velocity around S Per is $200 \mathrm{~km} / \mathrm{s}$.
\end{abstract}

Keywords. Astrometry, Masers, Supergiants.

\section{Introduction}

VLBI has a capability of determining positions of water masers relative to a phasereferencing calibrator with sub-milliarcsecond accuracy at short wavelengths (Hachisuka et al. (2006)). There are a number of stellar water maser sources in the circumstellar envelopes (CSE) of evolved stars, which are observable with VLBI at $22 \mathrm{GHz}$. The red supergiant star S Per is an interesting target for VLBI astrometry. Marvel (1996) estimated the distance to be $2.3 \pm 0.5 \mathrm{kpc}$ from the relative proper motions of the water maser features fitted to a symmetrically expanding outflow model. Phase-referencing VLBI for the star can directly detect absolute position changes of the maser features on the sky, which are very useful for investigating the annual parallax and proper motions. In addition, S Per is in the outer Galaxy so that it is also interesting for the study of the Galaxy's rotation curve. The Doppler shift of the maser emissions, and the transverse speed can be estimated from the proper motion and the distance determined from the annual parallax.

We have conducted VLBI phase-referencing observations of S Per relative to a closely located continuum source, KR143, with the VLBA at $22 \mathrm{GHz}$ over seven epochs (four years) since Nov 21st, 2000. We have also conducted phase-referencing observations of KR143 and ICRF $0244+624$ at $15 \mathrm{GHz}$ in the last four epochs to check the positional stability of KR143, which was selected from the 6-cm northern sky catalogue by Becker et al. (1991). Here we show the results of the annual parallax and proper motions of the water masers as well as the distance to S Per and the three-dimensional motion. The observed pairs of sources in our monitor program are listed in Table 1 with their separation angles and the observing frequencies. 
Table 1. Observed pairs of the sources in our monitoring program.

\begin{tabular}{|c|c|c|c|}
\hline Target & Positional reference & Separation angle & Observation frequency \\
\hline S Per & KR143 & $0.22^{\circ}$ & $22.2 \mathrm{GHz}$ \\
\hline KR143 & ICRF $0244+624$ & $4.8^{\circ}$ & $15.3 \mathrm{GHz}$ \\
\hline
\end{tabular}
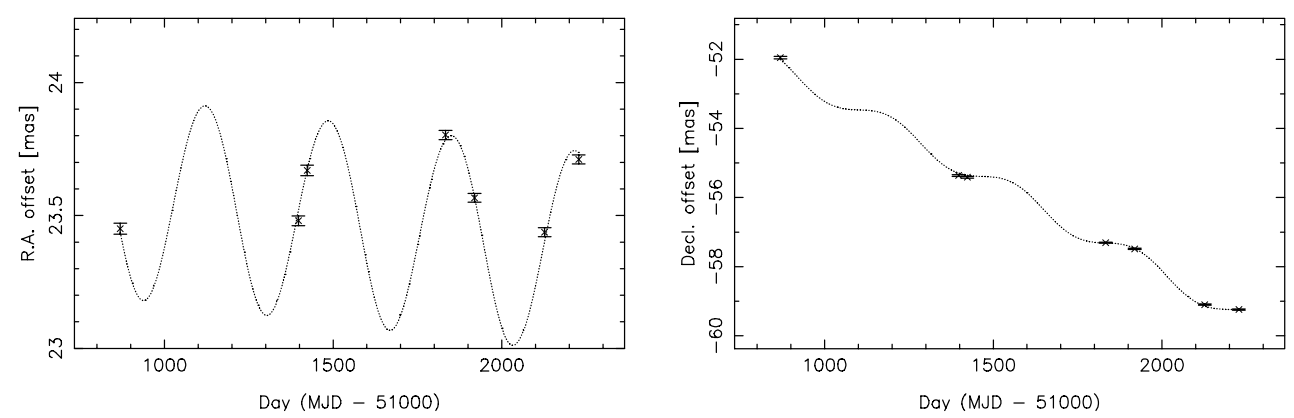

Figure 1. Maser feature motion relative to KR143. Dotted lines represent the best fit annual parallax and proper motion. The error bar is the root-square-sum of the position error of the maser feature and the assumed astrometric accuracy of $15 \mu$ arcsec.

\section{Results}

The position of KR143 relative to ICRF $0244+624$ was obtained with astrometric accuracy of 0.1 mas. If we take VLBI errors into consideration (the hydrostatic tropospheric delay error, the EOP error, and so on), this astrometric accuracy is appropriate for the $4.8^{\circ}$ separate pair of sources. The obtained KR143 positions at the four epochs do not show an indication of the proper motion and/or annual parallax. We then used KR143 as position reference for the phase-referencing astrometry of S Per. The astrometric accuracy of S Per is assumed to be $15 \mu$ arcsec for right ascension and declination.

A positional change of one of the maser features with the systemic velocity of $-42.0 \mathrm{~km} / \mathrm{s}$ is shown in Figure 1. The maser structure including this feature shows an arc-like structure expanding with time. This maser feature could be identified through all epochs. We fitted the motion of the maser feature to the simple model for annual parallax and proper motion. We estimated nine annual parallaxes. The averaged value and the standard deviation is $0.399 \pm 0.014$ mas $(2.51 \pm 0.09 \mathrm{kpc})$.

In the first three epochs, we identified 69 maser features. The proper motions is combined kinematics of the CSE and the central star. We fitted the proper motions to the expanding outflow model to obtain the proper motion of the central star, and the result is -0.38 and $-1.54 \mathrm{mas} / \mathrm{yr}$ for right ascension and declination, respectively. This proper motion is consistent with the Hipparcos result within its accuracy of 2 mas/yr. Assuming $R_{0}$ of $8.5 \mathrm{kpc}$ and $\Theta_{0}$ of $220 \mathrm{~km} / \mathrm{s}$, the distance from the Galactic center to $\mathrm{S}$ Per is $10.4 \mathrm{kpc}$. The estimated proper motion of the central star indicates that the Galactic rotation velocity around S Per is $200 \mathrm{~km} / \mathrm{s}$.

\section{References}

Becker, R. H., White, R. L., \& Edwards, A. L. 1991, ApJS 75, 1

Hachisuka, K., Brunthaler, A., Menten, K. M., Reid, M. J., Imai, H., Hagiwara, Y., Miyoshi, M., Horiuchi, S., \& Sasao, T. 2006, ApJ 645, 337

Marvel, K. B. 1996, PhD thesis, New Mexico State Univ., New Mexico. 


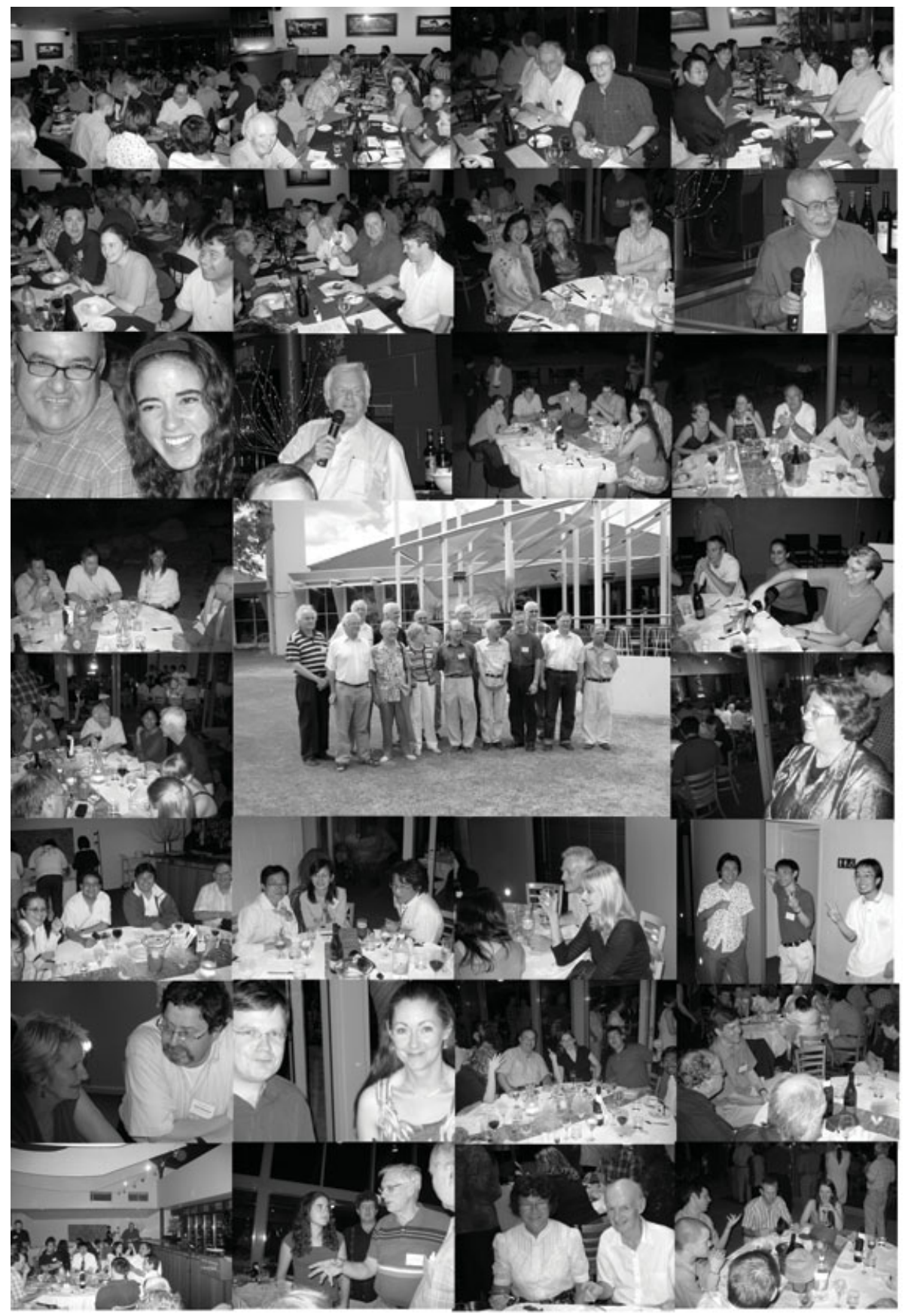

Photos: Miller Goss, Montage: Vicki Drazenovic 